\title{
Einleitung: Frequenzeffekte
}

Mit $>$ Rundfunkfrequenz $<$ oder mit $>$ Frequentieren $<$ hat dieser Band nicht direkt zu tun, sondern mit Häufigkeiten von Phänomenen, wie sie uns in der alltäglichen Erfahrung begegnen: Hört man beispielsweise eine Melodie immer wieder, kann sie einem unwillkürlich >nachlaufen<. Und umgekehrt: Hört man etwas Ungewöhnliches, Neues und Seltenes, zieht es unsere Aufmerksamkeit auf sich, da es hervorsticht.

In diesem Band soll es um Frequenzeffekte gehen, wie sie uns in der Sprache begegnen: Ungeachtet dessen, mit wem wir unsere Spracherfahrungen machen, ist eines klar: Es gibt Sprachformen, ja ganze Wortgefüge - auch Konstruktionen genannt -, die häufiger auftreten als andere. Wiederkehrende Strukturen begegnen uns in der Sprache >auf Schritt und ...Tritt $<$ : Eben dies ist eine Konstruktion, die wir für gewöhnlich nicht anders vervollständigen können. Einmal begonnen, kann die Konstruktion in der Regel nicht variiert werden.

Oder: Wie oft lernen wir, in einer Sprache ähnliche Elemente an die Stelle von anderen zu setzen: >Das gefällt mir sehr<, >das gefällt mir nicht<, >das gefällt mir ziemlich gut $<$, >das gefällt mir außerordentlich gut $<$. >Das gefällt $\mathrm{mir}+\mathrm{X}<$ ist einprägsam, da häufig: Einem Ohrwurm gleich? Nun, das Anfangsbild lässt sich nicht weiter strapazieren.

In den letzten Jahren hat sich in der Linguistik ein fruchtbarer Zweig entwickelt, der sich mit Gebrauchshäufigkeiten von sprachlichen Elementen (eben auch kompositer Natur) widmet: Umfangreiche Korpora vorab geschriebener Texte lassen sich heute, im Zeitalter der digitalen Schriftlichkeit, leicht nach Häufigkeiten von Elementen, ja nach ganzen Mustern, befragen. Verstehen wir häufige Kombinationen von Elementen schneller, wenn wir beispielsweise Texte lesen? Bewahrt die hohe Frequenz Elemente davor, dass sie obsolet werden? Werden beim kindlichen Spracherwerb besonders häufige Elemente leichter und deshalb früher erworben als andere? Dies sind einige der Fragen, denen man nachgehen kann. Frequenzeffekte wirken manchmal sehr direkt, wie zum Beispiel bei der artikulatorischen Reduktion von hochfrequenten Wortverbindungen. Neben dieser direkten Wirkung in vielen Bereichen - von der Syntax bis zur Diskursstruktur - gibt es jedoch auch Frequenzeffekte, die auf indirekte Weise wirkmächtig sind, da sie auf vermittelte Weise mit anderen Faktoren in den Bereichen Wandel, Verarbeitung und Erwerb interagieren.

Europäische Sprachen und Sprachvarietäten stehen bei diesen neuen Forschungsparadigma im Vordergrund, was vor allem der Verfügbarkeit von geeig- 
neten Korpora geschuldet ist. Korpora stehen dabei für Approximationen, die der Sprachwirklichkeit möglichst nahe kommen sollen. Denn diese Herangehensweise über die Analyse von Häufigkeiten ist notwendigerweise am konkreten Gebrauch der Sprache orientiert. Die gebrauchsorientierten Theorien gehen davon aus, dass die Häufigkeit eines linguistischen Phänomens in der mentalen Repräsentation von Sprache einen Niederschlag findet. Aktuelle Untersuchungen gehen über diese oft nur unterstellte Annahme hinaus, indem sie die Wirkung dieses Niederschlags untersuchen, und zwar in verschiedenen empirisch mit den Methoden der Linguistik und Kognitionswissenschaft erfassbaren Gebieten: im Sprachwandel, in der Sprachverarbeitung und im Spracherwerb. In diesem hohen empirischen und theoretischen Anspruch liegt das innovative Potential des Frequenzthemas.

Die nachfolgenden Beiträge sind teilweise im DFG-Graduiertenkolleg 1624 »Frequenzeffekte « und am Freiburger »Institute for Advanced Studies (FRIAS) « entstanden, teilweise kommen die Beiträge aus anderen Forschungszentren in Deutschland und den Niederlanden.

Der Nachweis von zahlreichen und unterschiedlichen Frequenzeffekten in den hier vorgestellten Forschungsarbeiten zeigt überzeugend, dass es eine statistische Dimension der menschlichen Erfahrung mit Sprache gibt. 